\title{
Metals Far From Equilibrium: From Shocks to Radiation Damage
}

E.M. Bringa, B.D. Wirth, M.J. Caturla, J. Stölken, D. Kalantar

This article was submitted to

$6^{\text {th }}$ International Conference on the Computer Simulation of Radiation Effects in Solids, COSIRES 2002, Dresden, Germany, June 23-27, 2002

U.S. Department of Energy

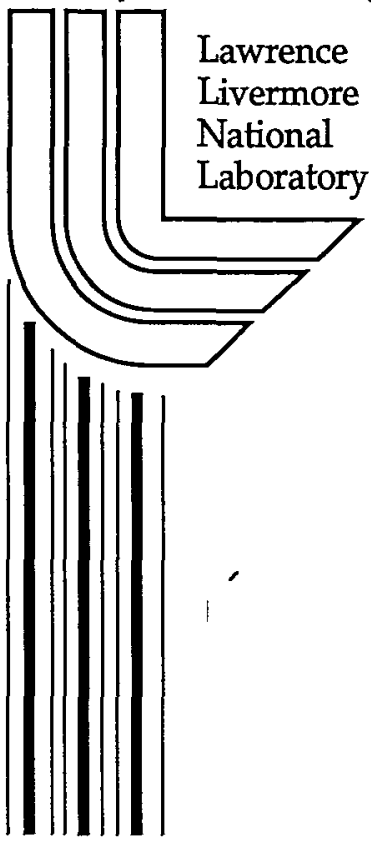

June 22, 2002 


\section{DISCLAIMER}

This document was prepared as an account of work sponsored by an agency of the United States Government. Neither the United States Government nor the University of California nor any of their employees, makes any warranty, express or implied, or assumes any legal liability or responsibility for the accuracy, completeness, or usefulness of any information, apparatus, product, or process disclosed, or represents that its use would not infringe privately owned rights. Reference herein to any specific commercial product, process, or service by trade name, trademark, manufacturer, or otherwise, does not necessarily constitute or imply its endorsement, recommendation, or favoring by the United States Government or the University of California. The views and opinions of authors expressed herein do not necessarily state or reflect those of the United States Government or the University of California, and shall not be used for advertising or product endorsement purposes.

This is a preprint of a paper intended for publication in a journal or proceedings. Since changes may be made before publication, this preprint is made available with the understanding that it will not be cited or reproduced without the permission of the author.

This report has been reproduced directly from the best available copy.

Available electronically at $\quad \mathrm{htp}: / / w w w . d o e . g o v / b r i d g e$

Available for a processing fee to U.S. Department of Energy and its contractors in paper from

U.S. Department of Energy

Office of Scientific and Technical Information

$$
\text { P.O. Box } 62
$$

Oak Ridge, TN 37831-0062

Telephone: (865) 576-8401

Facsimile: (865) 576-5728

E-mail: reports@adonis.osti.gov

Available for the sale to the public from

U.S. Department of Commerce

National Technical Information Service

5285 Port Royal Road

Springfield, VA 22161

Telephone: (800) 553-6847

Facsimile: (703) 605-6900

E-mail: orders@ntis.fedworld.gov

Online ordering: http://www.ntis.gov/ordering.htm

OR

Lawrence Livermore National Laboratory

Technical Information Department's Digital Library

http://www.llnl.gov/tid/Library.html 


\title{
Metals far from equilibrium:
}

\section{from shocks to radiation damage}

\author{
E. M. Bringa, B.D. Wirth, M.J. Caturla, J. Stölken, D. Kalantar ${ }^{1}$ \\ Lawrence Livermore National Laboratory \\ Chemistry and Material Sciences Directorate, MSTD \\ L353, PO. Box 808, Livermore, CA 94550
}

\begin{abstract}
Shock waves and high-energy particle radiation can each drive materials far from thermodynamic equilibrium and enable novel scenarios in the processing of materials. A large number of theoretical and experimental studies of shock deformation have been performed on polycrystalline materials, but shock deformation in single crystals has only recently been studied in some detail. We present Molecular Dynamics (MD) simulations of the shock response of single crystal copper, modeled using an embedded atom potential that reproduces both defect formation and high pressure behavior. Shock-induced plasticity will also be discussed. Predicting the in-service response of ferritic alloys in future fusion energy environments requires a detailed understanding of the mechanisms of defect accumulation and microstructure evolution in harsh radiation environments, which include a high level of He generation concurrent with primary damage production. The second half of this paper describes results of atomistic MD and kinetic Monte Carlo simulations to investigate the role of $\mathrm{He}$ on point defect cluster behaviour and damage accumulation in bcc Fe. The goal of these simulations is to study the mechanisms responsible for the formation of vacancy-He clusters which serve as He bubble and void nuclei in
\end{abstract}


fusion reactor materials.

Key words: shocks, plasticity, He bubbles

$P A C S$ :

\section{Introduction}

Ion irradiation of materials may take the target far away from equilibrium, leading to shock waves, nucleation of precipitates, phase changes, non-linear heat conduction, etcetera.

A number of scenarios show the importance of shock waves in ion bombardment. Simple shock models have been used to understand ejection following ion bombardment [1-3]. Simulations of cluster bombardment have shown hemispherical shock waves going inside the solid after cluster bombardment [4]. A similar situation appears for slow highly charged ion bombardment [5].In the electronic stopping regime, a cylindrical shock can arise as a result of the sudden heating of the ion track due to a number of repulsive events, like Coulomb explosion $[6,7]$. Using some model hydrodynamic simulations and $\mathrm{MD}$, it has been shown that this shock plays a very important role in track cooling and sputtering [8]. Laser ablation of material may also involve the production of a mainly planar shock waves, traveling away from the projectile [10]. As a result of the shock wave there may be solid-solid phase transitions and production of defects $[6,9]$. In the first part of this paper we use molecular dynamics (MD) simulations focus on the simplest possible geometry (planar shock) to study shock waves in metals, and to understand how the shock produces defects,

1 Corresponding author: tel. 1(925)423-5724; fax 1(925)423-7040; e-mail ebringa@llnl.gov 
changing the mechanical response of the material.

Among other radiation-induce processes which change the mechanical properties of the material, He nucleation and bubble formation in structural steels exposed to neutron irradiation can lead to shifts in the transition toughness curves (embrittlement) [11,12]. Yield and flow stress elevation (hardening) are also seen in these cases. The early stages of He bubble nucleation are not well understood and we use a combined MD and lattice kinetic Monte Carlo (LKMC) approach [12] to tackle this study, as explained in the second part of the paper.

\section{Shocks in single crystal cooper}

In order to understand atomistically some recent experimental results in single crystal $\mathrm{Cu}[14,15]$ we have done a number of MD simulations. Some details about the simulation are given below, together with results on the shock propagation.

\subsection{Molecular Dynamics Simulations}

Simulations were done with the code MDCASK [13], adapted to simulate shocks. An elongated box was equilibrated during several ps at $1.5 \mathrm{~K}$, using periodic boundaries in only the $\mathrm{x}$ and $\mathrm{y}$ directions. In this non-equilibrium MD simulations, a square pulse shock wave was applied along the $\mathrm{z}$ direction as follows. An extra force was added to a few planes of atoms (typically 2 planes) on one side of the sample. The force was kept constant throughout the simulation, although a time dependent profile could be applied. Velocity 
profiles were analyzed at different times giving both $U_{p}$ and $U_{s}$. There was a transient stage, lasting around 1 ps, which was neglected in the shock velocity calculations. Shock profiles were sharp, allowing for accurate determination of the shock speed. Both plastic and elastic fronts were seen in the simulations above a "plastic threshold". However, here we focus on the elastic shock.

Most non-equilibrium shock simulation studies to date have dealt with pair potentials, like Lennard Jones (LJ) [16-19], Morse, Buckingham, etcetera. Taylor and Dodson presented results on the Hugoniot of EAM Cu along <100> using an embedded-atom (EAM) potential, for $U_{p} \in(0,2.5)$ and a target with $4 \times 4 \times 30$ unit cells [20]. Here we use a EAM parametrization by Mishin and coworkers [21]. For shocks along $\langle 100\rangle$ most simulations reported here were done for sample sizes of $50 \times 50 \times 200 \mathrm{fcc}$ cells, i.e. $181 \times 181 \times 723 \mathrm{~nm}^{3}$ and 2 $10^{6}$ atoms. Some results were obtained for smaller samples with $25 \times 25 \times 100$ cells, and results for these two sample sizes were indistinguishable for the this potential. The following velocities will be given in $\mathrm{km} / \mathrm{s}$, unless noted otherwise. The initial temperature of the target was $2 \mathrm{~K}$, to avoid thermal noise in the shock propagation.

This EAM has been fitted to the cold curve of $\mathrm{Cu}$, a useful feature for the shock simulations at high pressure. It has a stacking fault energy $S T F E=44.0$ $\mathrm{mJ} / \mathrm{m}^{2}$, which compares well with experiments [21], and a cut-off radius, $r_{\text {cut }}$ after $4^{\text {th }}$ nearest neighbors (NN). It is important to point out that any pair potential with $r_{c u t}$ between $2^{\text {nd }}$ and $3^{\text {rd }}$ nearest neighbors will have $\mathrm{STFE}=0$, and therefore dislocations invariably appear as partials moving as independent dislocations with no binding force due to the stacking fault. On the other hand, $S F T E>0$ results in a finite separation between the partial dislocations in the fcc crystal, namely around $2 \mathrm{~nm}$ for this EAM potential. The lateral size 
of the target has to be able to accommodate this separation, and therefore at least 6 unit cells are required in the lateral direction for the potential used here.

\subsection{Shock front and plasticity}

When the piston is moved above a certain pressure two shock fronts are produced. The first one is the elastic front, which is also produced at lower pressures, and that only compresses the lattice creating few point defects due to large fluctuations near this front. The second one is the plastic front, which needs some threshold stress vale to be initiated, same as in the perfect LJ crystal [16].

Fig. 1 shows a snapshot from the MD simulation of a $50 \mathrm{GPa}$ shock, moving in the direction of the arrow, 7.8 ps after the shock started. Only a fraction of the sample is shown. Size of the atoms is proportional to their kinetic energy, $E_{k i n}$. Color is also proportional to $E_{k i n}$, with "colder" atoms blue and "hotter" atoms red. The position of the elastic and plastic shock fronts are shown, with the plastic front about ten crystal planes behind the elastic front.

Figure 2 shows what happens when there is no plastic front produced. The elastic "wave-train" has been seen in LJ crystals simulations at $0 \mathrm{~K}$ [16]. The "ringing" of the velocity profile is produced by adjacent planes hitting each other back and forth. This effect is produced by the ABAB stacking of $\{100\}$ planes.

From the velocity profiles at different times one can calculate both piston velocity, $U_{p}$, and the shock velocity, $U_{S}$. In this case the shock pressure is 
$P_{H}=20 \mathrm{GPa}$, and $U_{S}=(4.56 \pm 0.1) \mathrm{km} / \mathrm{s} ; U_{p}=(0.44 \pm 0.01) \mathrm{km} / \mathrm{s}$. Fig.

3 , on the other hand shows what happen for very strong shocks. The elastic front is almost $100 \%$ engulfed by the plastic front $\sim 3.2$ ps after the shock was turned on. In this case the crystal behind the shock is molten, but the shock is propagating into a pristine crystal.

At pressures above elastic behavior shown in Fig. 2 and below the shock melting in Fig. 3, the plastic ve nucleates dislocations as it travels trough the pristine lattice. As a result, and due to the separation of partial dislocations by stacking faults (STF), a criss-crossing network of STF's is observed behind the shock front. Figure 4 shows this resulting defect structure, which is also seen in simulations using a short range LJ potential, where the STF energy is zero. The atoms in STF's are highlighted by using a potential energy filter. This is the same frame of Fig. 1, for a $50 \mathrm{GPa}$ shock. The formation of stacking faults has been identified as the main dislocation multiplication mechanism near the shock front by a recent model [22].

All the above results are for an initially perfect crystal, with no defects. However, even well annealed "single crystal" metals will have a residual dislocation density. We are currently studying the influence of defects on the shock proagation and induced plasticity. Among those possible deffects, one could include voids and bubbles, which are the topic of the following sections.

\section{Dynamics of $\mathrm{He}$ in $\mathrm{Fe}$}

Radiation damage in both fission and fussion reactors could modify the mechanical properties of structural steels. Substitutional $\mathrm{Cu}$ precipitate forma- 
tion and evolution has been studied with some detail, using both MD [23] and KMC techniques [12]. One of the key ingredients in the damage processes is He cluster formation. Althoug there are many atomistic studies on small He clusters in fcc metals like $\mathrm{Ni}$ [24], the role of $\mathrm{He}$ in bcc metals has not been studied in detail by atomistic methods with few exceptions, among them the classical work of Wilson and Johnson [25], who studied substitutional and interstitial $\mathrm{He}$ in bcc Fe.

In order to understand initial stages of $\mathrm{He}$ cluster/bubble nucleation at times much greater than seconds, the following approach is used to track the fate of all defects, including He. First, a MD database of displacement cascades which gives the primary defect production [26] is built. Defect clustering and kinetics can be further studied using MD as explained below. A short-time ( 100 ns) Defect kinetic Monte Carlo (DKMC) [27], off-lattice, is used to allow additional recombination and self-interstitial atoms (SIA) clustering and migration away from the cascade. The resulting configuration is the input of our LKMC simulation.

\subsection{Molecular Dynamics Simulations}

We used a modification of MDCASK by K. Morishita [28]. The interaction potentials for Fe-Fe was the Finnis-Sinclair potential from Ackland [29], with a cut-off radius $\mathrm{rCut}=3.76 \AA$ (includes second $\mathrm{NN}$ ). The Fe-He potential we used is a fit to ab-initio calculations of small He-Fe clusters by Wilson ( $\mathrm{rCut}=3.80$ $\AA$ ) [30]. For the He-He potential we use the Beck potential [31], with two changes: a) it is splined to a ZBL potential at high energy $(\sim 25 \mathrm{eV})$; b) it is splined to a smooth exponential cutoff $(\mathrm{rCut}=3.80 \AA)$ in order to reduce the 
original cut-off from the Beck potential ( $\mathrm{rCut}=11.00 \AA$ ) to something more tractable computationally. We have checked that the spline cut-off did not introduce any spurious effects for small He clusters. However, some longer range contributions could be needed if the He density inside a bubble is. From now on $\mathrm{V}$ designs vacancy sites, $\mathrm{HeS}$ design $\mathrm{He}$ substitutional sites, and $\mathrm{HeI}$ designs He interstitials.

In order to run the LKMC, the energies at the saddle point along migration routes are needed. Using MD, saddle point energies were calculated as follows. He was placed at a certain position and not allowed to move. Then a simulation at $T=1000 \mathrm{~K}$ was run, which was quenched to $0 \mathrm{~K}$ during approximately $5 \mathrm{ps}$. The total energy of the system was calculated for different He positions along certain symmetric trajectory and the energy at the saddle point was obtained as the highest energy barrier along the trajectory. From previous studies of HeI migration, the energy barrier is $0.078 \mathrm{eV}$ [28]. For HeS to switch with a vacancy is $0.12 \mathrm{eV}$ and finally for a $\mathrm{V}$-Fe jump the barrier is $0.9 \mathrm{eV}$ [12]. We have calculated also the saddle energy for HeS-2NNV jump and it is $0.66 \mathrm{eV}$. Allowing the He to move freely one can study different migration processes. For instance, substitutional He migrates to a vacant site along the $<111>$ direction.

Below we will discuss the LKMC approach, where all atoms are located in at a perfect lattice. However, one may ask if it is valid to use a lattice approach when there are interstitials that deform the equilibrium lattice. We have used $\mathrm{MD}$ simulations to study the lattice deformation due to small He clusters. Initial $\mathrm{He} / \mathrm{V} \gtrsim 10$ give raise to loop punching of interstitials, and $\mathrm{He} / \mathrm{V} \gtrsim 5$ gives raise to significant lattice deformation. However, for He/V smaller than 5 the LKMC approach would be justified. Fig. 5(a) shows a $\mathrm{He}_{6} \mathrm{~V}_{2}$ complex, which 
only causes some lattice relaxation, but does not punch any SIA. The figure shows all Fe atoms within a cube of side $2 a_{o}$ centered at the He cluster, which is roughly ellipsoidal. Fig. 5(b) shows the He atoms only. $\mathrm{A} \mathrm{He}_{20} \mathrm{~V}_{1}$ complex, which decays into a $\mathrm{He}_{20} \mathrm{~V}_{13}$ complex by SIA punching is shown in Fig. 6(a). If the evolution of this complex had been done at low temperatures, the SIA would be preferentially arranged on one side of the cluster forming a SIA loop, as it has been observed in MD simulations of $\mathrm{He}$ in Ni. However, at $\mathrm{T}=1000$ $\mathrm{K}$ the thermal fluctuations seem to destroy the symmetry of the process and the SIA loop is not formed. In order to avoid any temperature effects in the final analysis, we quench the system down to $T<10^{-3} \mathrm{~K}$ during few ps. Then the displacement field is calculated using the quenched configuration. The size of the Fe atoms in Fig. 6(b) is proportional to their radial displacement from their initial lattice positions and shows some large anisotropies. This would mean that for these $\mathrm{He} / \mathrm{V}$ ratios one would need to use an off-lattice $\mathrm{KMC}$ approach that includes the role of stress in the diffusion energies. Fig. 6(b) shows that the He cluster has a rough fcc packing.

\subsection{Lattice Kinetic Monte Carlo Simulations}

We adapted a LKMC code that has been successfully used to study Cu precipitates in $\mathrm{Fe}$ [12]. Cu was considered only substitutional, but He can start out as an interstitial or in a substitutional site. Based on the MD simulations, we only had to add a sub-lattice including all the octahedral sites of the bcc lattice. Tetrahedral sites are unstable along migration paths. Our LKMC simulations uses the same potentials used in the MD evaluated at lattice sites. He-He interactions are included up to 5th NN. Fe interstitial has a high mobility and one expects any produced interstitials to rapidly migrate to interstitial 
sinks.

All atoms can only jump to NN sites. Since HeI has low migration energy and $\mathrm{HeS}$ has large binding energy to $\mathrm{V}$, a loop over all HeI is performed first, until there are no HeI left or no V left where the HeI could move to. Second, a loop over all vacancies is run, allowing exchanges with $\mathrm{Fe}$ and $\mathrm{HeS}$ atoms. Probabilities are re-normalized by highest probability of any jump, setting the effective time scale, which changes because probabilities depend on local environment. At least one jump is always accepted, and the remaining possible jumps are accepted or discarded following a Metropolis scheme. This scheme used to aprove jumps needs an energy barrier for a given move, which depends on the local environment and it is taken to be $\Delta E=E_{\text {saddle }}+\frac{1}{2} \Delta E_{\text {inzt-final }}$, where $E_{\text {saddle }}$ is given above, and $\Delta E_{\text {init-final }}$ is the energy difference of the system before and after the jump.

The boundaries of the box act as vacancy sinks. When a $\mathrm{V}$ arrives to the boundary, it is transformed into a Fe atom. Fig 7 shows one snapshot at 0.1 $\mu$ s from a LKMC run. Only V, HeS, and HeI sites are shown. Initial vacancy distribution was obtained from an "aged" $20 \mathrm{keV}$ cascade, with the addition of $0.05 \%$ residual $\mathrm{V}$, and $0.1 \% \mathrm{He}(2 / 3$ as $\mathrm{HeS})$, making $92 \mathrm{~V}, 51 \mathrm{HeS}$ and $26 \mathrm{HeI}$ in a box with $40 \times 32 \times 30$ bcc cells $(307,200$ total sites). Only $73 \mathrm{~V}$ and 5 HeI were left when the snapshot was taken. Each HeI is forming a dimer with a HeS. The lifetime of these dimers is typically few ns. Note that without the spatial concentration of $\mathrm{V}$ due to the collision cascade the diffusion of HeS would be greatly diminished. After $1 \mathrm{~ms}$, all vacancies were eliminated by migration to the boundary, and the He left show several dimers and couple trimers. In this example, the number of displacements per atom were dpa $=$ $\left(\mathrm{V} / \mathrm{N}_{b c c-s i t e s}\right)=1.210^{-3}$. After $10^{4} \mathrm{~s}$, the dose rate would be $1.210^{-7} \mathrm{dpa} / \mathrm{s}$, 
in the appropriate range for fussion reactors.

\section{Summary}

Shocks provide a way to study localized high strain and strain rate in materials. Shock induced deformation of material near ion tracks has already been proposed to induce damage in metallic alloys. With the advent of better accelerators for cluster bombardment of solids, large energy densitites and momentum transfer could be achieved, and shock effects would arise.

In this paper we considered planar shocks in single crystal $\mathrm{Cu}$ at different pressures. At relatively low shock pressures (below $~ 30 \mathrm{GPa}$ ), only an elastic front is seen, while at higher pressures a plastic front arises, moving faster than the elastic front and overtaking it. This plastic front leaves behind a material with defects. $<100>$ shocks in EAM Cu show plastic deformation through stacking faults. This behavior seems to be insensitive to the election of a two body or a many body potential to perform the simulation. We are int he process of studying the plasticity mechanisms for single crystals along other crystalline directions, were LJ potentials already predict some rich behavior. We are also including defects like voids and $\mathrm{He}$ bubbles to asses the influence of the initial microstructure in the final, post-shock, microstructure.

We developed a Lattice Kinetic Monte Carlo code to track insterstitial He , substitutional He, and vacancies, to perform atomistic studies on the initial stages of He bubble nucleation in bcc metals. We do find some rapid formation of He dimers and trimers. More details on the He clustering process and on dose and dose rate effects are in progress. Based on future results on should 
be able to produce cross-sections which could improve current rate models of bubble nucleation [32]. Among other possible studies one could consider influence of precipitates ( $\mathrm{Cu}, \mathrm{Cr}, \mathrm{C}$, etc.) and extended defects (dislocation, grain boundaries, surfaces) on He migration. The stress induced by precipitates/defects may change the migration energies resulting in different final $\mathrm{He}$ cluster distribution.

\section{Acknowledgements}

This work was performed under the auspices of the U. S. Department of Energy by the University of California, Lawrence Livermore National Laboratory under Contract No. W-7405-Eng-48. E.M. Bringa would like to thank K. Morishita for helpful discussions.

\section{References}

[1] Y. Kitazoe, N. Hiraoka and Y. Yamamura, Surf. Sci. 111, 381 (1981).

[2] D. Fenyo and R. E. Johnson, Phys. Rev. B 46, 5090 (1992).

[3] I. S. Bitensky and E. S. Parilis, Nuc. Inst. Meth. B 21, 26 (1987).

[4] Z. Insepov et al., Phys. Rev. B 61, 8744 (2000).

[5] M. Hedström and H-P. Cheng, Phys. Rev. B 62, 2751 (2000).

[6] D. Lesueur and A. Dunlop, Rad. Eff. and Def. in Solids 126, 123 and 163 (1993).

[7] E.M. Bringa and R.E. Johnson, Phys. Rev. Lett. 88, 165501 (2002).

[8] M. Jakas, E.M. Bringa and R.E. Johnson, Phys. Rev. B 65, 165425 (2002). 
[9] V. P. Zhukov and A. V. Ryabenko, Rad. Effects 82, 85 (1984).

[10] L. L. Zhigilei and B. Garrison, Proceedings of Laser Tissue Interaction IX, S. Jacques et al eds., (1998), page 135.

[11] G.R. Odette et al., MRS Bulletin 26, 176 (2001).

[12] G. Odette and B. D. Wirth, J. Nuc. Mat. 251, 157 (1997).

[13] T. Diaz de la Rubia and M. W. Guinan, Mat. Res. Forum 174, 151 (1990).

[14] D. H. Kalantar et al., Phys. Plasmas 7, 1999 (2000).

[15] A. Loveridge-Smith et al., Phys. Rev. Lett. 86, 2349 (2000).

[16] B. L. Holian and P. S. Lomdahl, Science 80, 2085 (1998).

[17] V. V. Zhakhovskii, S. V. Zybin, K. Nishihara, and S. I. Anisimov, Phys. Rev. Lett. 83, 1175 (1999).

[18] T. C. Germann, B. L. Holian, P. S. Lomdahl, and R. Ravelo, Phys. Rev. Lett. 84, 5351 (2000).

[19] J.-B. Maillet, M. Mareschal, L. Soulard, R. Ravelo, P. S. Lomdahl, T. C. Germann, and B. L. Holian, Phys. Rev. E 63, 016121 (2001).

[20] P.A. Taylor and B. W. Dodson, Phys. Rev. B 42, 1200 (1990).

[21] Y. Mishin, M. J. Mehl, D. A. Papaconstantopoulos, A. F. Voter and J. D. Kress, Phys. Rev. B 63, 224106 (2001).

[22] E. Zaretsky, J. App. Phys. 78, 3740 (1995).

[23] J. Marian, Phys. Rev. B 65, 144102 (2002).

[24] J. B. Adams and W.G. Wolfer, J. Nuc. Mat. 158, 25 (1988).

[25] W. D. Wilson and R.A. Johnson, in Interatomic Potentials and Simulation of Lattice Defects, Eds. P. Gehlen, J.R. Beeler and R. I. Jaffe, Plenum Press, 1972, page 375 
[26] This cascades, generated by a primary knock-on atom of few tens of keV's were provided by R.E. Stoller, ORNL.

[27] B. D. Wirth et al, Nuc. Inst. Meth. B 180, 23 (2001).

[28] K. Morishita et al, The fourth Pacific Rim International Conference on Advanced Materials and Processing, Ed. by S. Hanada et al., The Japan Institute of Metals, 2001. page 1395.

[29] G.J. Ackland et al., Phil. Mag. A 75, 713 (98).

[30] W. D. Wilson, private communication.

[31] D. E. Beck, Mol. Phys. 14, 311 (1968).

[32] R. E. Voskoboinikov and A. E. Volkov, J. Nuc. Mat. 297, 262 (2001). 


\section{FIGURES}

Fig. 1. Snapshot from the MD simulation of a $50 \mathrm{GPa}$ shock, moving in the direction of the arrow, $7.8 \mathrm{ps}$ after the shock started. Only a fraction of the sample is shown, but the view goes trough all 100 [010] planes. Size of the atoms is proportional to their kinetic energy, $E_{k i n}$. Color is also proportional to $E_{k i n}$, with colder atoms blue and hotter atoms red. The position of the elastic and plastic shock fronts are shown.

Fig. 2. 20 GPa shock. Velocity versus depth at three different times. There is a train of elastic waves and there is no evidence of a plastic front.

Figure 3. $300 \mathrm{GPa}$ shock. Velocity versus depth at four different times. The elastic front is being overtaken by a plastic front.

Fig. 4. Same shock from Fig. 1. Atom color proportional to potential energy. Plastic deformation occurs by formation of stacking faults, seen as the crisscross pattern in the figure.

Figure 5. Snapshots of a $\mathrm{V}_{2} \mathrm{He}_{6}$ complex from $\mathrm{MD}$ simulations at $1000 \mathrm{~K}$. (a) . All Fe atoms within a cube of side $2 a_{o}$ centered at the He cluster are shown. There are no extra vacancies produced. (b) only the He cluster is shown.

Figure 6. Snapshots of a $\mathrm{V}_{1} \mathrm{He}_{20}$ complex from $\mathrm{MD}$ simulations at $1000 \mathrm{~K}$, slowly quenched to $0 \mathrm{~K}$ after $10 \mathrm{ps}$. For $\mathrm{He} / \mathrm{V}$ ratios greater than 10 , interstitial punching occurs (12 new interstitials in this case), creating a $\mathrm{V}_{13} \mathrm{He}_{20}$ complex. Only the interstitial $\mathrm{Fe}$ atoms are shown, with a size proportional to their radial displacement. (b) The He cluster shows fcc-like packing.

Figure 7. Snapshot at $0.1 \mu$ s from a LKMC run. Only V, HeS, and HeI sites 
are shown. Initial state: $\mathrm{V}$ from $20 \mathrm{keV}$ cascade, $0.05 \%$ residual $\mathrm{V}$, and $0.1 \%$ He $(2 / 3$ as HeS). Only $73 \mathrm{~V}$ and $5 \mathrm{HeI}$ were left when the snapshot was taken. 


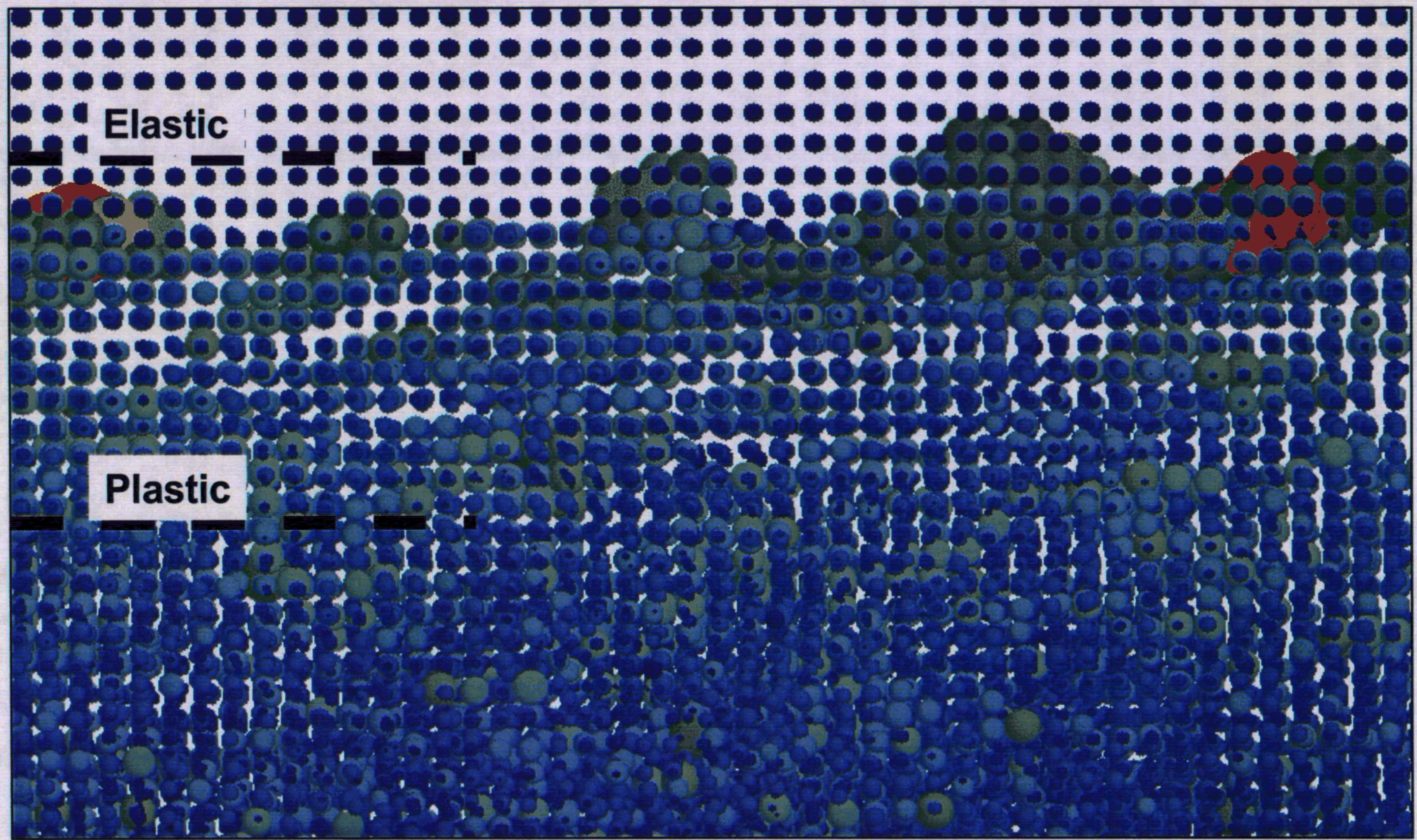

Fig 1. Bringa et al. 


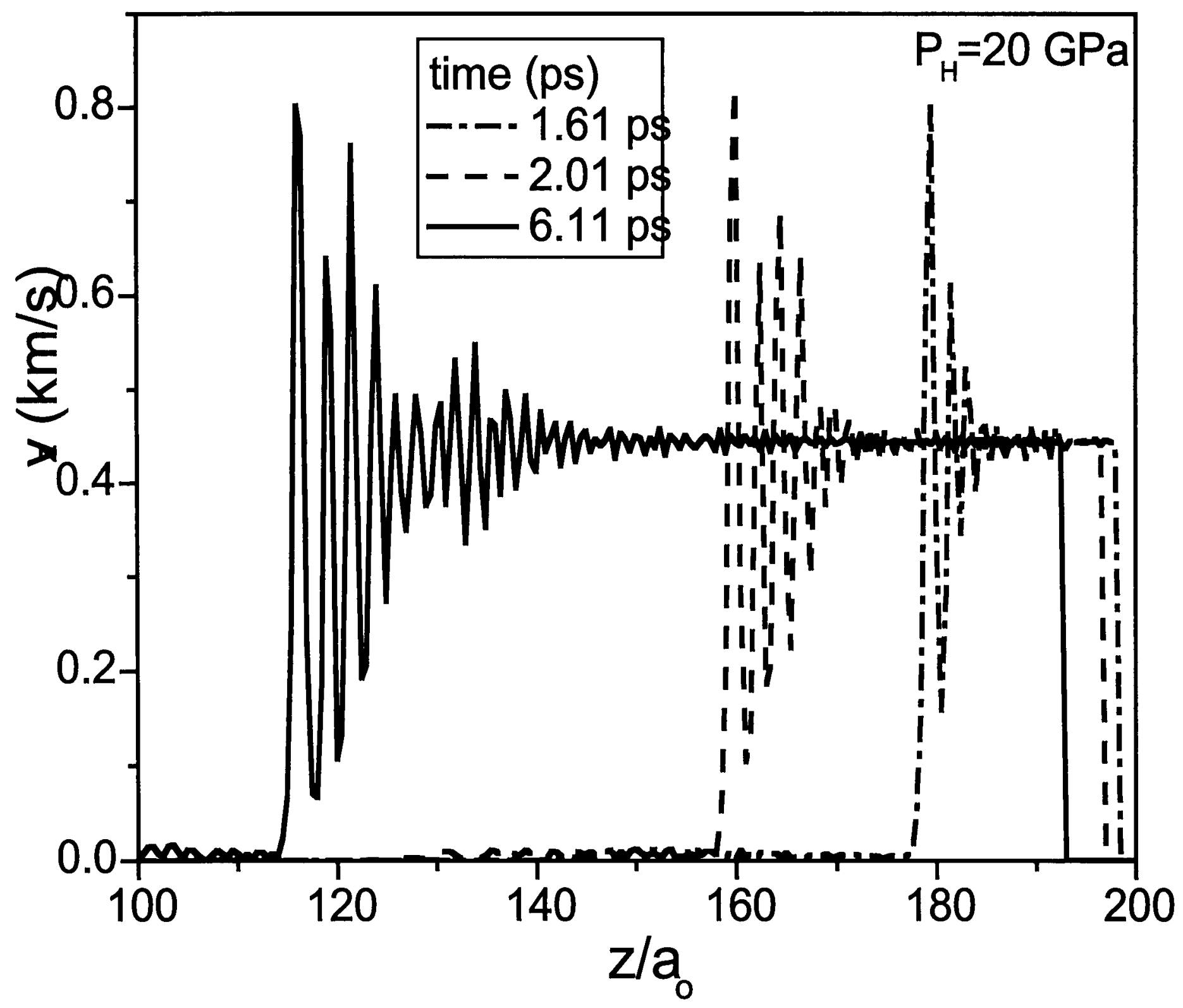

Fig. 2. Bringa et al. 


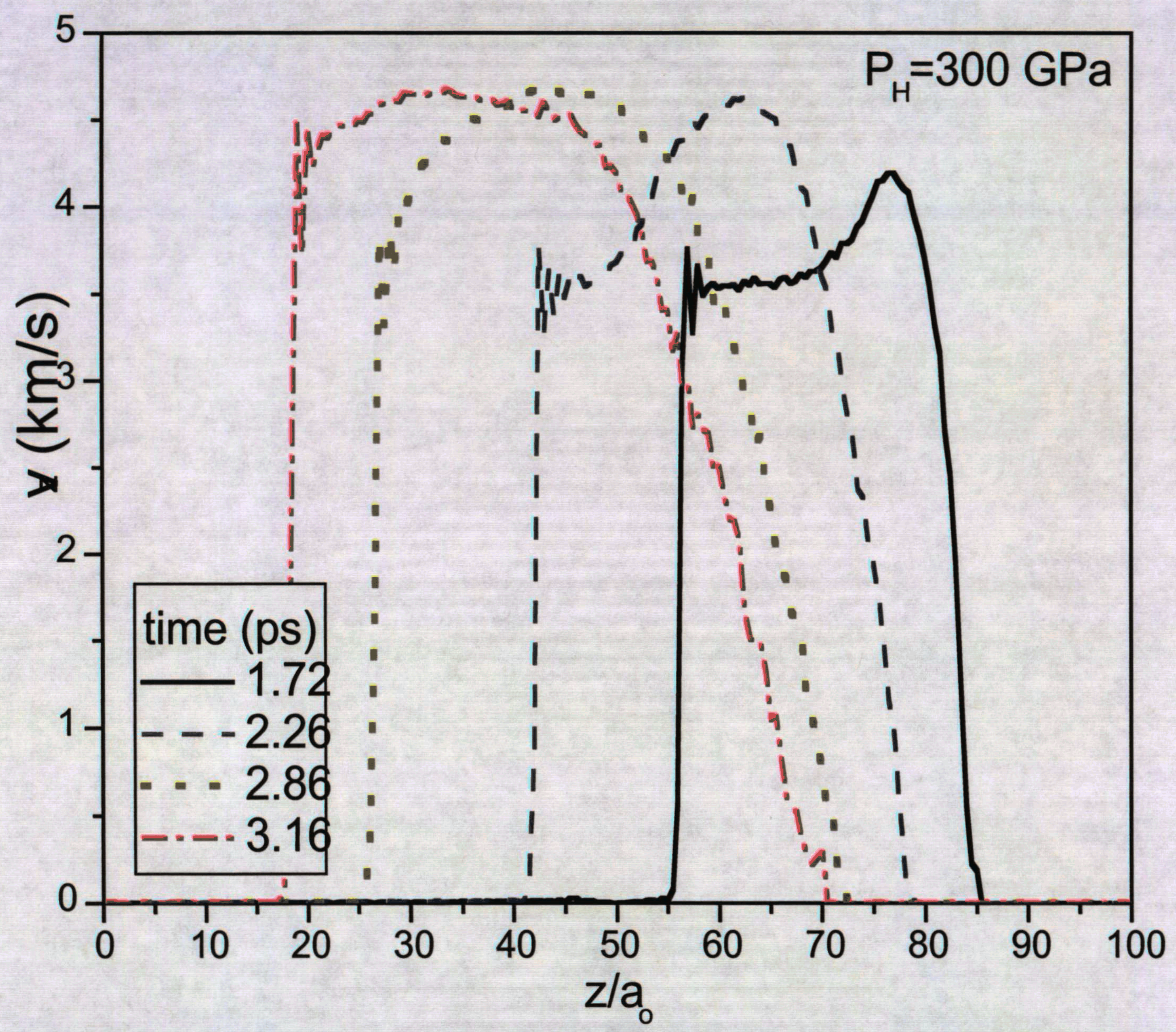

Fig. 3. Bringa et al. 


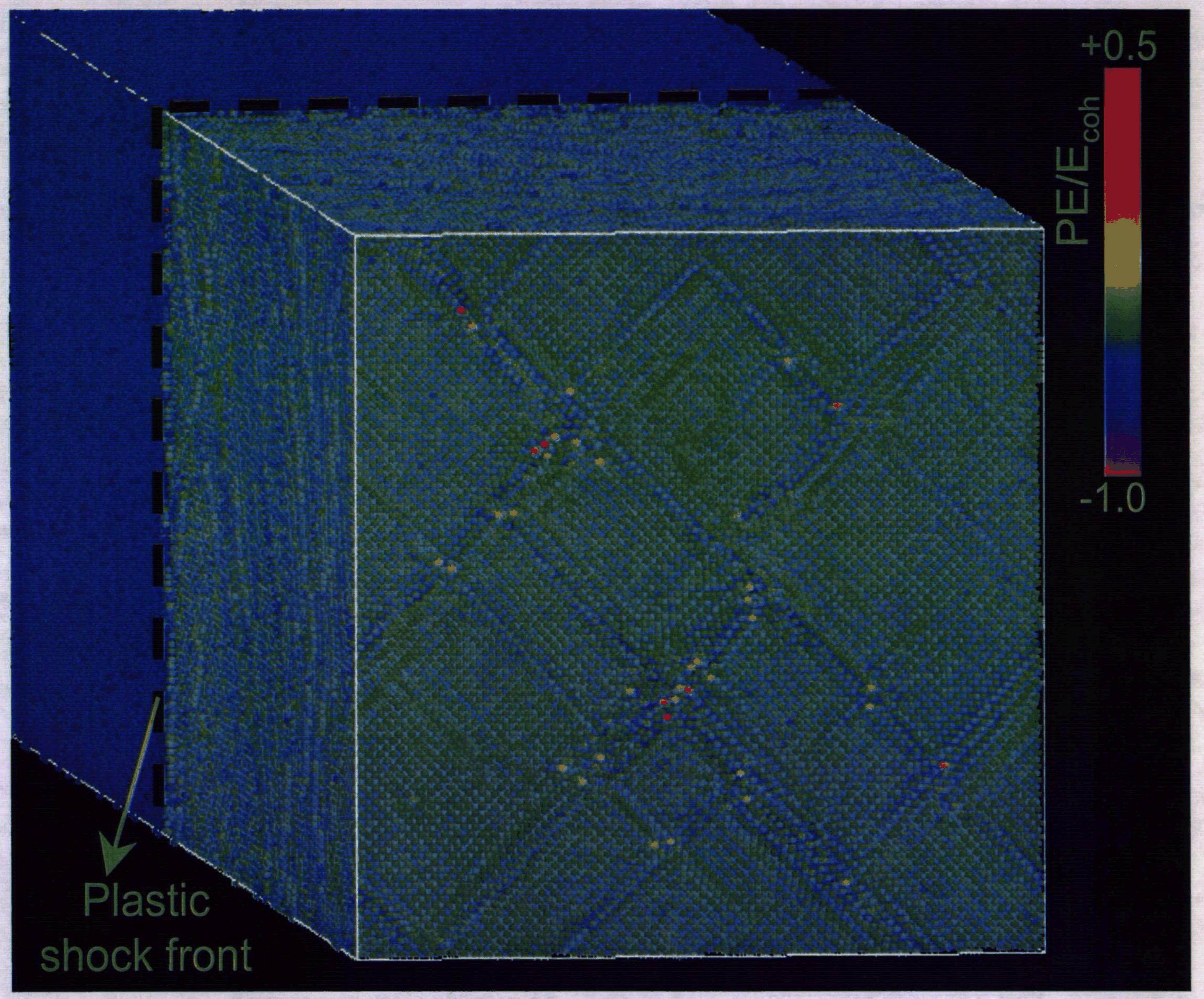

Fig 4. Bringa et al. 


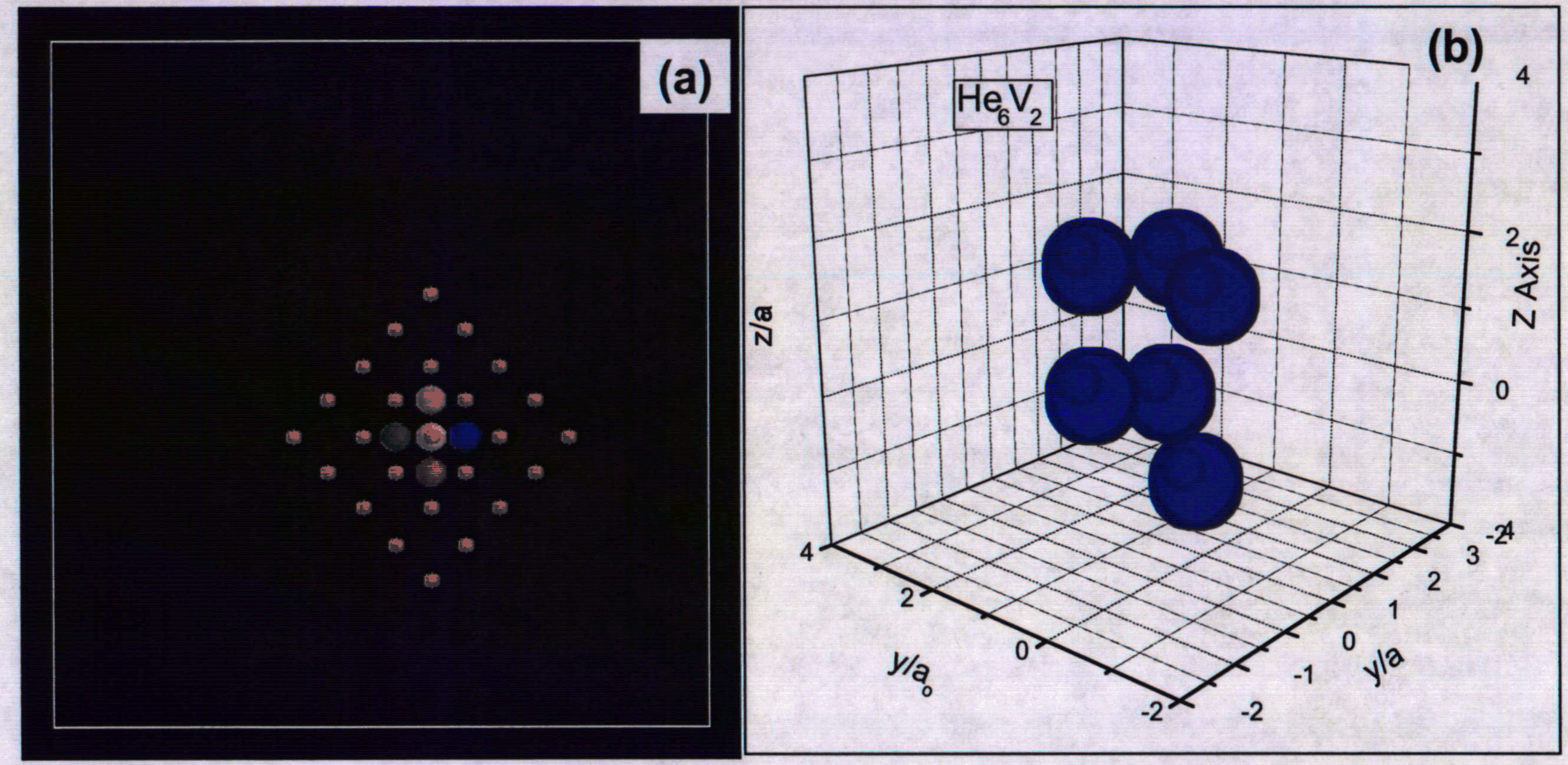

Fig 5. Bringa et al. 


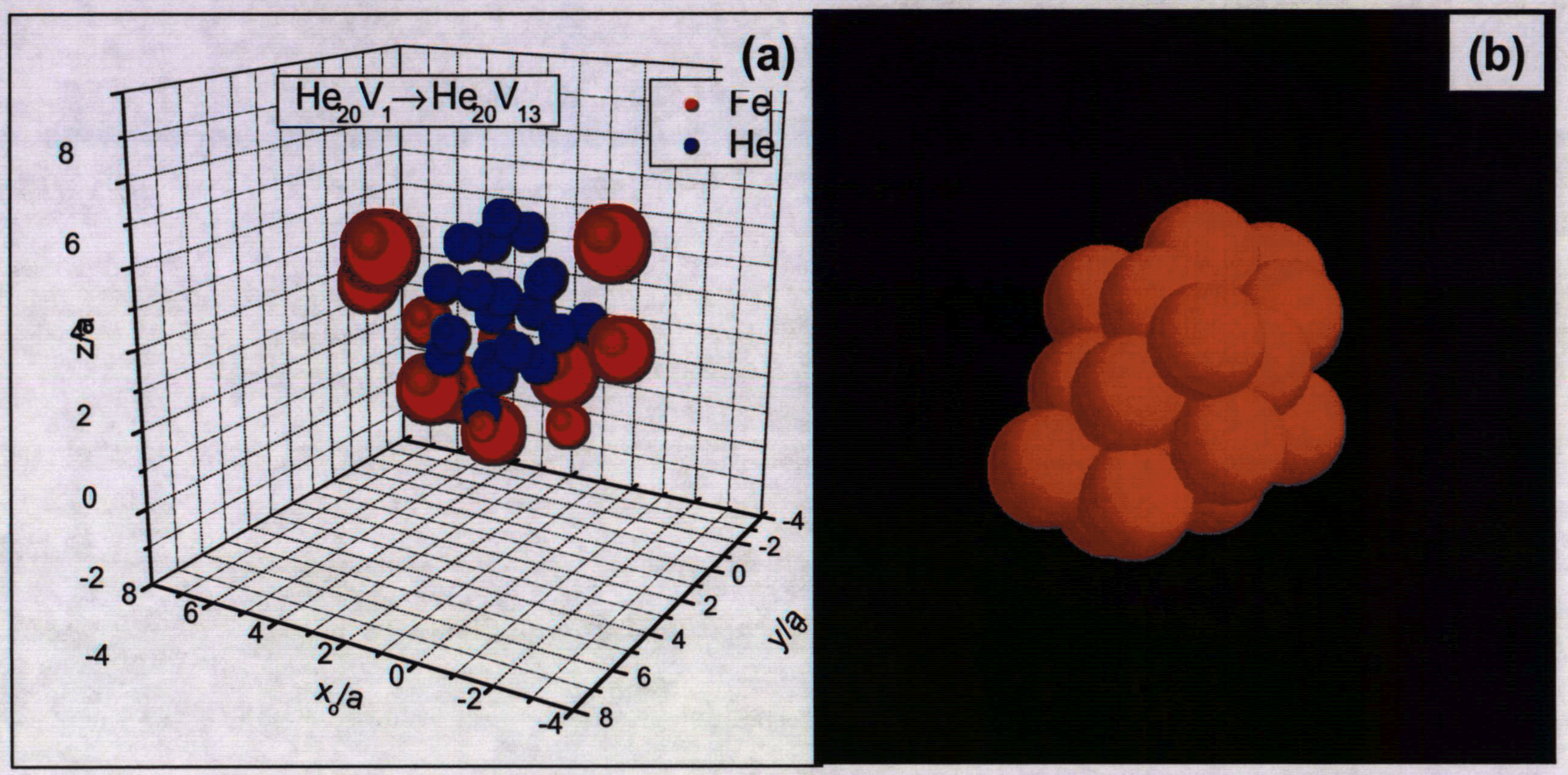

Fig 6. Bringa et al. 


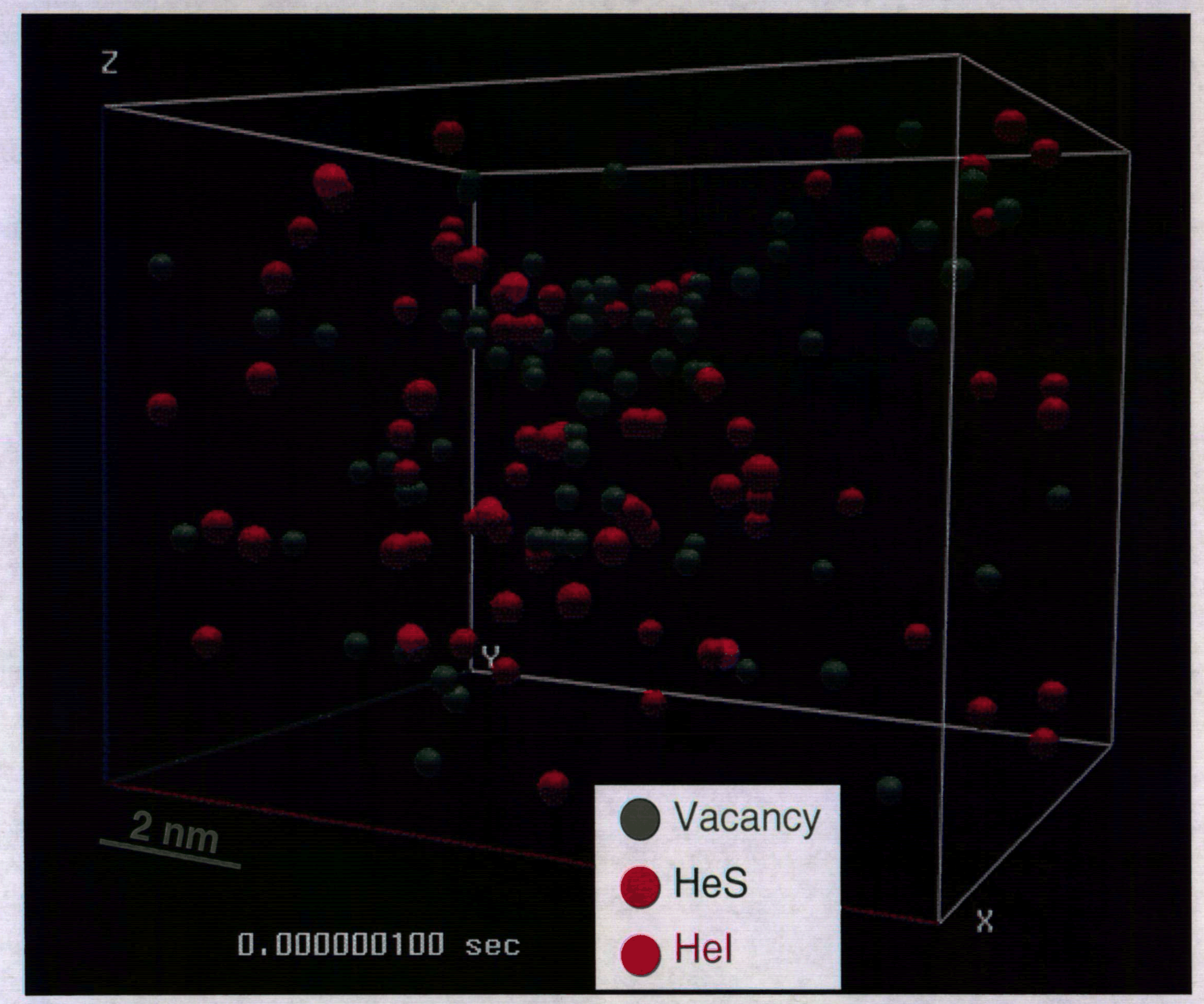

Fig 7. Bringa et al. 